\title{
An Analysis Framework for Bank Capital Allocation*
}

\author{
Nicolas Baud Antoine Frachot Philippe Igigabel \\ Pierre Martineu Thierry Roncalli \\ Groupe de Recherche Opérationnelle \\ Crédit Lyonnais
}

December 1, 1999

\begin{abstract}
In this paper, we present a methodology for capital adequacy. Capital adequacy in a bank could be viewed as an asset allocation problem. Therefore it is possible to use the framework of the portfolio theory. But it requires to estimate the correlation matrix of the business line returns. In this work, we estimate this correlation matrix by using a factor model based on external data. In a second part of this article, we provide some illustrations to rebalance optimally the capital allocation within the bank.
\end{abstract}

\section{Introduction}

Capital allocation within a bank is getting more important as the regulatory requirements are moving towards economic-based measures of risk. Banks are urged to build sound internal measures of credit and market risks for all their activities. In this context, capital allocation is a crucial task which is very similar to a portfolio management problem. More precisely, a top-down approach consists in disaggregating a bank's portfolio into different business lines with different ratios of expected return and different risks. Then the capital should be allocated in order to balance the portfolio in an optimal way.

According to the portfolio theory, a bank has to evaluate not only the expected return and the risk of every business line, but also the correlation matrix of these business lines' returns. In most cases, the bank has a good knowledge of its expected returns and risks. As for the correlation matrix, the problem is more complex: the bank does not have enough internal data and information to estimate it accurately. In this respect, we develop an approach based on a Market Factor Model: we estimate an implied correlation matrix using the returns of a panel of banks. The second section presents this model.

The third section deals with the allocation problem. An asset allocation problem cannot exactly stand for a capital allocation problem. Nevertheless the portfolio theory can be adapted so that it takes capital allocation's specific issues into account. For example, a bank has to abide by some solvency rules, it also has to reach some implicit objectives fixed by its shareholders. Besides, capital allocation could be viewed as an optimisation problem or an economic utility function problem under specific constraints.

Actually, the allocation problem is not exactly the problem a bank has to cope with. A bank is caracterised by its initial allocation. Moving to a new and better allocation generates costs that can be taken into account by penalizing the utility function. Nonetheless, if such a method determines the position of the optimum, it does not exhibit any path going from the initial allocation to the optimal one. Then, we introduce reallocation signals allowing a dynamic policy that leads to the optimum.

*We gratefully thank Jean-Yves Rossignol, Patrick Amat, Andrée-Lise Rémy as well as the Capital Allocation team at Crédit Lyonnais for stimulating discussions. All remaining errors are ours. To send us your comments, contact Antoine Frachot (antoine.frachot@creditlyonnais.fr) or Thierry Roncalli (thierry.roncalli@creditlyonnais.fr). 
This paper is based on two works made at the Operational Research Group of Crédit Lyonnais ([7], $[15])$. The findings being confidential, this paper is not extensively illustrated by figures. Moreover, we have decided to conceal any reference to particular financial institutions. Yet, the main lines have been preserved.

\section{A market factor model}

Let's consider a bank organized into $M$ business lines. Let $f_{t}^{(m)}$ be the return of the business line $m$ and $\alpha_{t}^{(m)}$ be the proportion of capital allocated to this business line at the period $t$. The bank's return $r_{t}$ is assumed to be equal to

$$
r_{t}=\sum_{m=1}^{M} \alpha_{t}^{(m)} f_{t}^{(m)},
$$

which means that the total return of the bank amounts to the sum of the business lines' returns. We can impose that $f_{t}^{(m)}$ does not depend on the bank so that $f_{t}^{(m)}$ is assimilated to a factor, and the asset class factor model (SHARPE [1992]) is

$$
r_{t}=\sum_{m=1}^{M} \alpha_{t}^{(m)} f_{t}^{(m)}+u_{t}
$$

This model is quite classical and it can be traced back to the Asset Pricing Theory from Ross [1976]. In the equation (2), $u_{t}$ is the "non-factor" component of the return. One of the main problem lies in determining the values of the unknown factors $f_{t}^{(m)}$. Nevertheless, in a context of gaussian asset allocation, the problem can be solved if the the factors' first and the second moments are known.

Using this model, we can estimate the correlations between the different business lines' returns. Then, this "external" correlation matrix can be used in an internal model based on top-down methodology. Let's point out that such a model is not perfect: an "internal" correlation matrix would be more relevant but, most of the time, a bank lacks internal data to estimate it acurrately. The approach above gets rid of this problem.

\subsection{Assumptions and statistical properties of the model}

Let's consider a panel of $N$ banks. Let $r_{t, n}$ be the observed market return of the bank $n$ at the period $t$. Let $\alpha_{t, n}=\left(\alpha_{t, n}^{(m)}\right)$ denote its sensitivity vector at the same period

$$
\alpha_{t, n}=\left[\begin{array}{c}
\alpha_{t, n}^{(1)} \\
\vdots \\
\alpha_{t, n}^{(m)} \\
\vdots \\
\alpha_{t, n}^{(M)}
\end{array}\right]
$$

The statistical model can be written as

$$
r_{t, n}=\alpha_{t, n}^{\top} f_{t}+u_{t, n}
$$

where $f_{t}=\left(f_{t}^{(m)}\right)_{1 \leq m \leq M}$ is the random vector of the business lines' returns and $u_{t, n}$ an indiosyncratic term which captures all the specificities of the bank $n$. Since $f_{t}$ does not depend on the bank, $\alpha_{t, n}^{\top} f_{t}$ can be viewed as the market composant of the stock return. The residual $u_{t, n}$ takes into account the fact that two banks with similar capital allocations does not necessarily have the same stock returns. As a consequence, we naturally suppose that the $u_{t, n}$ are statistically uncorrelated between two banks and also serially uncorrelated. Moreover, we suppose that $f_{t}$ and $u_{t}$ are independant and we assume that $f_{t} \sim \mathcal{N}(\mu, \Sigma)$ and $u_{t, n} \sim \mathcal{N}\left(0, \sigma_{n}^{2}\right)$. Then, we have

$$
\begin{aligned}
\mathbb{E}\left[r_{t, n}\right] & =\alpha_{t, n}^{\top} \mu \\
\operatorname{var}\left[r_{t, n}\right] & =\alpha_{t, n}^{\top} \Sigma \alpha_{t, n}+\sigma_{n}^{2}
\end{aligned}
$$


It becomes apparent that the variance of the bank's return is the sum of two components. The first component is a factor risk common to every bank. Two banks with the same capital allocation have the same factor risk. Let's notice that this factor risk is a lower risk bound $\left(\operatorname{var}\left[r_{t, n}\right] \geq \alpha_{t, n}^{\top} \Sigma \alpha_{t, n}\right.$ ). The second component is a specific risk that can be viewed as an efficiency measure of the management of the bank.

\subsection{The estimation method}

With a panel of $N$ banks, the matrix form of the relationship (4) is

$$
\mathbf{r}_{t}=A_{t} f_{t}+\mathbf{u}_{t}
$$

where $r_{t}=\left(r_{t, n}\right)$ is the $N \times 1$ vector of returns, $A_{t}=\left(\alpha_{t, n}^{\top}\right)$ the $M \times N$ matrix of sensitivities and $u_{t}=\left(u_{t, n}\right)$ the $N \times 1$ vector of residuals. Let $D$ denote the covariance matrix of $u_{t}$. To simplify the problem, we suppose that there is no correlation between the specific components of two different banks. Then, we have

$$
D:=\operatorname{diag}\left(\begin{array}{ccccc}
\sigma_{1}^{2} & \cdots & \sigma_{n}^{2} & \cdots & \sigma_{N}^{2}
\end{array}\right)
$$

The log-likelihood function of the model (7) is easy to derive in a gaussian framework but it still provides unbiased estimates under alternative statistical assumptions (see Gourieroux, Monfort and TrognON [1984]). This property is useful since returns can hardly be considered as gaussian. Given $T$ dates of observation, the pseudo log-likelihood function is

$$
\begin{aligned}
\ell(\mu, \Sigma, D \mid \mathbf{r}) & \equiv \sum_{t=1}^{T} \ell_{t}\left(\mu, \Sigma, D \mid \mathbf{r}_{t}\right) \\
& =-\frac{N T}{2} \ln 2 \pi-\frac{1}{2} \sum_{t=1}^{T} \ln \left|A_{t} \Sigma A_{t}^{\top}+D\right|-\frac{1}{2} \sum_{t=1}^{T}\left(r_{t}-A_{t} \mu\right)^{\top}\left(A_{t} \Sigma A_{t}^{\top}+D\right)^{-1}\left(r_{t}-A_{t} \mu\right)
\end{aligned}
$$

Let $\theta=(\mu, \Sigma, D)$ be the parameter vector. The maximum likelihood estimate is defined as the solution of the following optimisation problem

$$
\begin{array}{r}
\hat{\theta}=\arg \max \ell(r \mid \mu, \Sigma, D) \\
\text { u.c. }\left\{\begin{array}{l}
\Sigma>0 \\
D>0
\end{array}\right.
\end{array}
$$

This problem could be solved numerically using a quasi-Newton algorithm like BFGS.

Remark 1 If we assume that the matrix $A_{t}$ is time-independant, then the score vector of the concentrated log-likelihood is

$$
\mathbf{g}\left(\theta^{c} \mid \mathbf{r}\right)=-\left[\begin{array}{c}
\operatorname{vech}\left(T A^{\top}\left(\Omega^{-1}-\Omega^{-1} \hat{V} \Omega^{-1}\right) A H\right) \\
\operatorname{Diag}\left(T \Delta\left(\Omega^{-1}-\Omega^{-1} \hat{V} \Omega^{-1}\right)\right)
\end{array}\right]
$$

with

$$
\theta^{c}=\left[\begin{array}{c}
\operatorname{vech} H \\
\operatorname{Diag} \Delta
\end{array}\right]
$$

$\Omega$ corresponds to $A H H^{\top} A^{\top}+\Delta^{2}$ and the matrices $H$ and $\Delta$ are defined such that $H$ is the lower triangular Cholesky decomposition of $\Sigma$ and $\Delta$ is the squared root matrix of $D$. $\hat{V}$ is the empirical $N \times N$ covariance matrix of the returns $\mathbf{r}_{t}$. We could also determine $\hat{\theta}_{\mathrm{ML}}^{c}$ as the solution of the likelihood equations $\mathbf{g}\left(\theta^{c} \mid \mathbf{r}\right)=\mathbf{0}$. And $\hat{\mu}_{\mathrm{ML}}$ is defined as $A^{\dagger} \hat{\mathbf{r}}$ with $A^{\dagger}$ the Moore-Penrose pseudo-inverse of $A$ and $\hat{\mathbf{r}}$ the empirical mean vector of the returns.

Remark 2 We are interested in the correlation matrix $\rho$ of the business lines' returns. Let's point out that the $M L E$ of $\rho$ is very easy to compute once $\Sigma$ or $H$ are estimated.

Remark 3 Since the number of parameters is very high ( $\left.\operatorname{dim} \theta^{c}=N+\frac{M(M+1)}{2}\right)$, starting values are very important for the optimisation convergence. We note that we could easily compute them using the OLS (or $G L S)$ estimates of the model (7). 


\subsection{Data description}

In order to perform the estimates, we first have to specify the data we need to define the model inputs:

1. The annual report may be used to define the returns $r_{t, n}$. Yet, in this case, the number of observations is not large enough to obtain consistency in ML estimation. So we have taken the daily stock returns from january 1994 to july 1999.

2. As for the factors $f_{t}^{(m)}$, we decompose the banking activity into 8 typical business lines: retail banking, middle-market, asset mangement, corporate banking, structured finance, corporate market, life insurance and non-life insurance. The model can be applied to our whole panel or separetely, to different geographic areas. In this work, we have defined a European area and an American one. Then, we obtain 16 different factors.

3. The main issue lies in determining the different banks' sensitivities. We may assume that they are related to the current capital allocations. In this respect, each bank's capital allocation was conveniently treated to obtain a consistent (but probably approximated) measure of the economic capital dedicated to each business line. This hard task was performed by our Crédit Lyonnais expert, J-Y. Rossignol.

Secondly, we built a panel of financial institutions with KMV Monitor. The panel contains 315 banks, insurance and financial institutions. We encountered some problems with asian banks so that we decided to leave them out of the panel. As a consequence, we defined only two geographic areas. Moreover, some of the banks' stock returns presented particularities because of fusion, capital modifications and so on. Finally, we obtained a panel of 173 financial institutions. The geographic decomposition of our panel is as follows:

\begin{tabular}{|l|c|}
\hline Germany & 22 \\
France & 24 \\
United Kingdom & 20 \\
Italy & 15 \\
Spain & 8 \\
Netherlands & 6 \\
Swiss & 5 \\
Other european countries & 18 \\
\hline United States & 55 \\
\hline
\end{tabular}

The following table exhibits the overall allocation in Europe and in the United States:

\begin{tabular}{|l|c|c|c|}
\hline Business lines & Europe & United States & Total \\
\hline Retail banking & $16 \%$ & $28 \%$ & $20 \%$ \\
Middle-market & $22 \%$ & $22 \%$ & $22 \%$ \\
Asset mangement & $6 \%$ & $5 \%$ & $6 \%$ \\
Corporate banking & $15 \%$ & $7 \%$ & $12 \%$ \\
Corporate market & $11 \%$ & $17 \%$ & $13 \%$ \\
Structured finance & $5 \%$ & $6 \%$ & $6 \%$ \\
Non-life insurance & $13 \%$ & $10 \%$ & $12 \%$ \\
Life insurance & $12 \%$ & $5 \%$ & $10 \%$ \\
\hline
\end{tabular}

\subsection{Econometric results}

In order to preserve the confidentiality of our findings, we do not present them exhaustively. Moreover, we renamed randomly the business lines.

Some interesting results comes out of the estimated values. For example, the European correlation matrix presents some coefficients that are very different from the American ones. Thus, the correlation coefficient value between the business lines BL \#1 and BL \#4 is 0.83 for the United States and -0.34 for Europe! It also appears that some European correlation coefficients are non significant as it is the case for 
$\rho(\mathrm{BL} \# 1, \mathrm{BL} \# 2)$. Finally, we may point out that the cross-correlations between business lines in Europe and in the United States are smaller than the correlations observed separetely in both geographic areas, but only one is negative!

As an illustration, we exhibit the correlation matrix between the 8 American business lines:

\begin{tabular}{|lcccccccc|}
\hline \hline & BL \#1 & BL \#2 & BL \#3 & BL \#4 & BL \#5 & BL \#6 & BL \#7 & BL \#8 \\
\hline \hline BL \#1 & 1.00 & 0.63 & 0.57 & 0.83 & 0.87 & 0.91 & 0.87 & 0.80 \\
BL \#2 & & 1.00 & 0.31 & 0.69 & 0.44 & 0.64 & 0.60 & 0.46 \\
BL \#3 & & & 1.00 & 0.41 & 0.73 & 0.45 & 0.68 & 0.62 \\
BL \#4 & & & & 1.00 & 0.53 & 0.94 & 0.75 & 0.71 \\
BL \#5 & & & & 1.00 & 0.69 & 0.75 & 0.68 \\
BL \#6 & & & & & 1.00 & 0.76 & 0.73 \\
BL \#7 & & & & & & 1.00 & 0.91 \\
BL \#8 & & & & & & & & 1.00 \\
\hline \hline
\end{tabular}

After estimating the worldwide correlation matrix, we performed its principal component analysis, which leads to the following results. With the first seven components, we obtain a cumulative quality of about $95 \%$. The first component reflects a worldwide trend factor (we may notice that almost all the business lines are represented in a same proportion). The second component is a geographic component: we clearly observe an opposition between European and American business lines. Nonetheless, three business lines do not take part in the elaboration of this axis (European BL \#1 and American BL \#2 and BL \#3). The third component shows a link between the three European activites BL \#1, BL \#2 and BL \#4. The fourth component is an american axis and concerns the activites BL \#2, BL \#3, BL \#4 and BL \#5. The remaining components oppose specific European activities to other specific American activities.

Moreover, the model presented here is interesting because the equation (6) gives the decomposition of the risk of a bank between a factor-market risk and a specific risk. We can evaluate the proportion $\kappa_{n}$ of the risk explained by the factor-market risk. With the ML estimate, $\kappa_{n}$ takes the following value

$$
\kappa_{n}=\frac{\alpha_{n}^{\top} \hat{\Sigma} \alpha_{n}}{\alpha_{n}^{\top} \hat{\Sigma} \alpha_{n}+\hat{\sigma}_{n}^{2}}
$$

Thus, it is possible to classify the financial institutions according to $\kappa_{n}$. The graphic (1) represents the histogram of $\kappa_{n}$. We observe that in general, European banks have a smaller $\kappa$ than American banks. That probably indicates that stock returns contains more information in American markets than in European ones.

Until now, we only considered the correlation matrix between the different business lines' returns. Let's point out that the model also permits to estimate the expected returns and risks. In an internal approach of capital allocation, these informations does not seem very useful. Internal expected returns and risks of every business line would be more relevant. Nethertheless, the estimates based on our panel stress out European and American specific behaviours. In the figure (2), we performed a mean-variance analysis. European business lines are generally less risky that American ones, but in the same time have smaller expected returns. We computed the efficient portfolios that are given by the traditional mean-variance optimisation $\operatorname{program}^{1}$ (MARKOWITZ [1987]):

$$
\begin{array}{ll}
\sup _{\alpha} & \alpha^{\top} \mu \\
\text { u.c. } & \left\{\begin{array}{l}
\alpha^{\top} \Sigma \alpha \leq \mathrm{s} \\
\alpha^{\top} \mathbf{1}=1
\end{array}\right.
\end{array}
$$

The solution of the program (14) is the efficient frontier (every combination of portfolios belonging to this frontier remains efficient). We clearly note that the European frontier is on the left of the American one,

\footnotetext{
${ }^{1}$ The first constraint $\alpha^{\top} \Sigma \alpha \leq \mathrm{s}$ can be interpreted as a regulatory requirement similar to a value-at-risk requirement.
} 


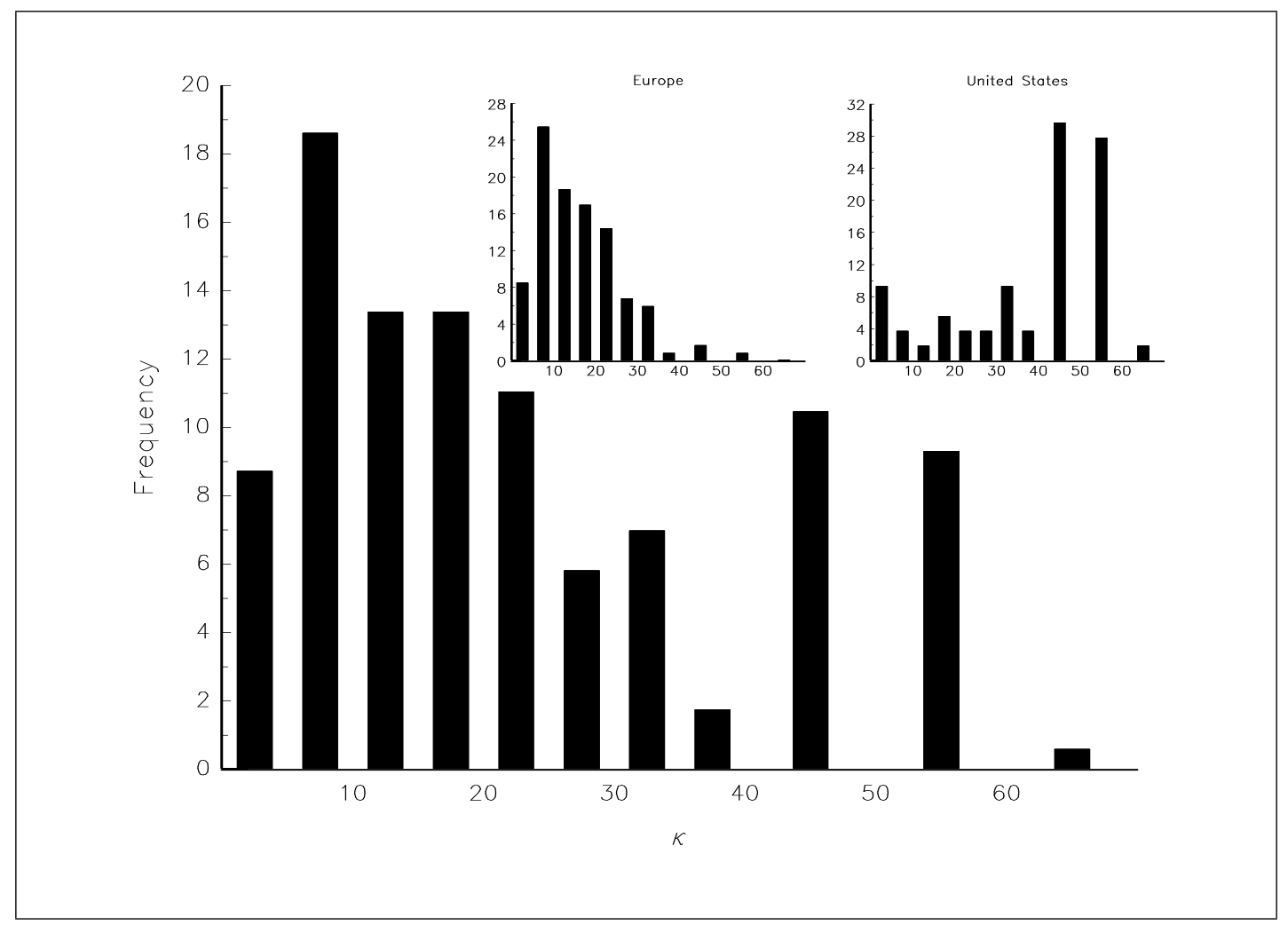

Figure 1: Proportion of risk explained by the factor-market risk

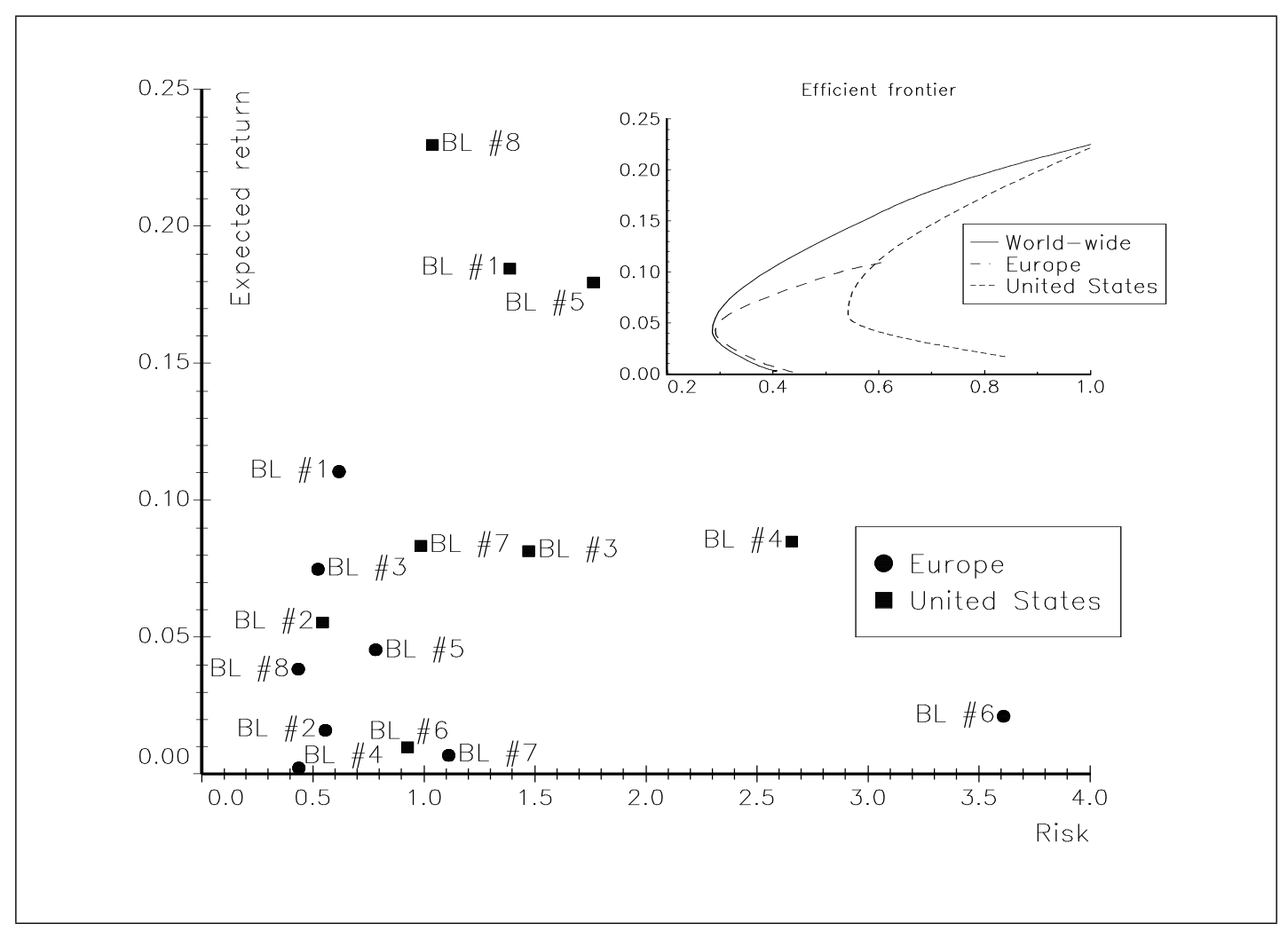

Figure 2: Mean-Variance analysis 
which illustrates the previous remark. Such a result may be explained by the difference of growth trend observed between Europe and the United States.

If we go back to the main point at issue, we now dispose of an estimated correlation matrix which allows to implement a top-down approach of the internal capital allocation,

\section{The capital allocation problem}

In this section, we try to define the capital allocation problem. In a first paragraph, we introduce the concept of internal model and the interest of such a tool. In a second paragraph, we show that a capital allocation problem is not as simple as portfolio management. Finally, we provide an example using the correlation matrix we estimated.

\subsection{Building an internal model}

We consider an objective function that has to be maximized. In corporate finance, this function is the firm value, more precisely the shareholder's wealth. The Shareholder Value Added analysis (SVA) consists in defining the rules permitting to reach this objective. One problem is to define exactly the shareholder's wealth and its measure. We could for example simplify the problem by utilizing the Market Value Added (MVA) which is equal to the firm's total maket value minus the capital invested. This shareholder value concept is directly derived from market capitalisation. At this point, we must consider the following problem: it is not certain that stock prices accurately reflect the value of the firm. That's why we refer to the shareholder utility function without any specific reference to a value added measure or a shareholder wealth measure. Actually, the shareholder utility function is the core of any capital allocation internal model.

An internal model for capital allocation must be based on a methodology. In portfolio management, we traditionally distinguish among two methods. The first method, called top-down, consists in allocating capital to different asset classes, and then selecting the best securities within each asset class. In the second method, the bottom-up approach, we directly select the best securities. These two methods are now being used in bank capital allocation, but with some differences (reference [2]) because capital allocation systems in banks are based on the notion of Economic Capital: we could define it as the amount of capital appropriate to cover worst-case losses in all but the most extreme economic scenarios.

The report [2] shows that "the banks reviewed by the Task Force utilize internal economic capital allocations for two broad purposes: measuring risk-adjusted profitability, and portfolio risk management". The bottom-up approach is relevant in the first case whereas the top-down approach is adapted to portfolio management. A top-down internal allocation model can be viewed as a portfolio problem, the portfolio consisting of the bank's business lines.

The model uses two different categories of data. Internal data provide us with the expected returns and the risks of the bank's business lines. External data are employed to estimate the correlation matrix as defined in the methodology above. The expected returns and the risk measures could be completed by performance objectives announced by the different business lines. Let's notice that data collection is an important step and one has to be careful with the numbers provided by an historical analysis.

Moreover, the internal model has to take regulatory requirements into account. Another constraint concerns strategic issues, the general orientation of the bank's policy. Eventually, the solution given by the model is bound to depend on the current allocation. Indeed we do not deal with an allocation model but a reallocation one, which generates specific costs.

The figure (3) sums up the internal model process. 


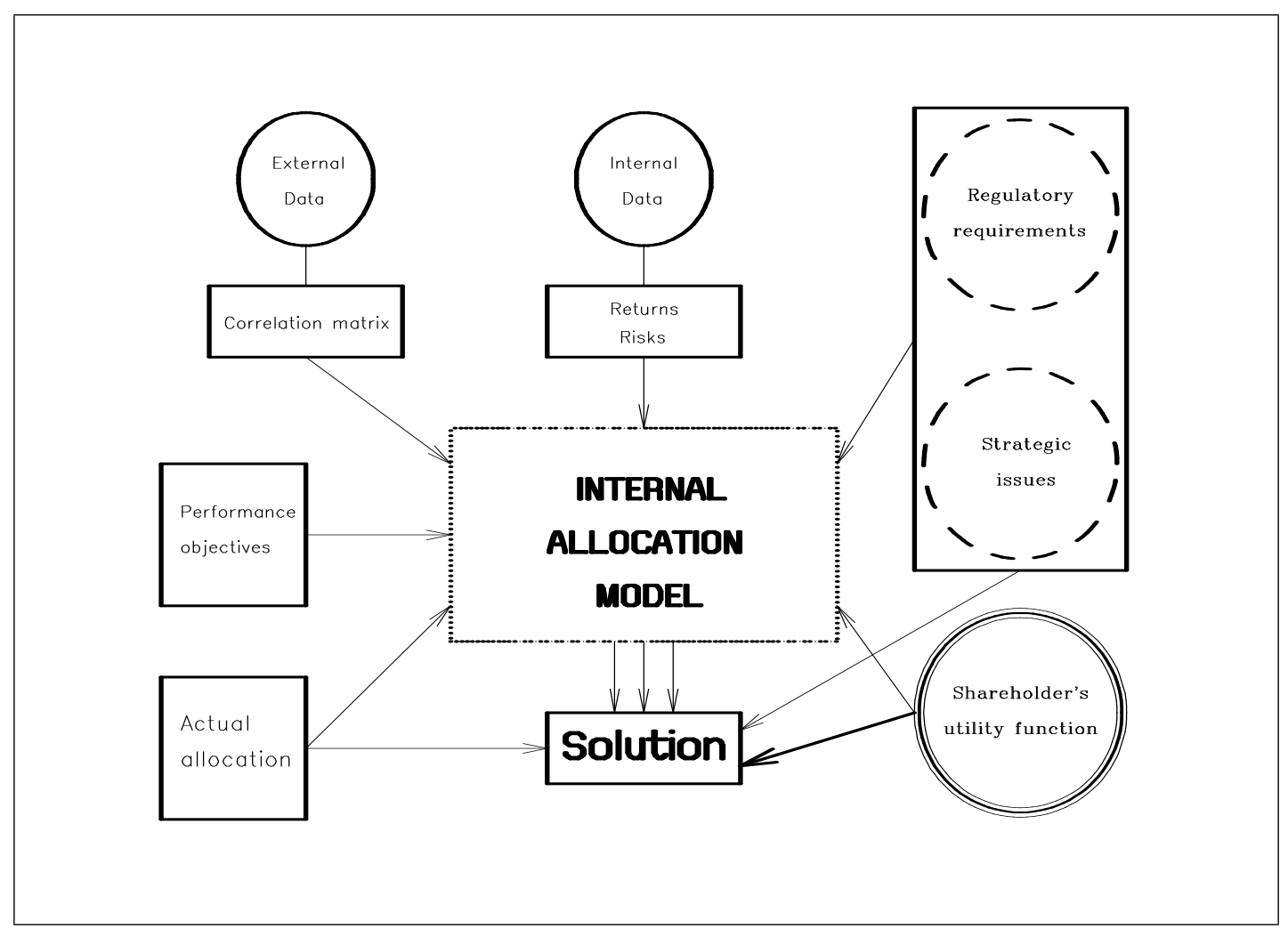

Figure 3: Internal model building scheme

\subsection{Mathematical setting}

Let $\alpha$ denote the allocation vector of the $M$ business lines. The internal allocation model can be formulated as an optimisation problem

$$
\begin{aligned}
\alpha^{\star}= & \arg \max f(\alpha ; \mathcal{I}) \\
& \text { u.c. } \quad \alpha \in \Omega
\end{aligned}
$$

where $f$ is the objective function. In a SVA approach, this objective function is linked (directly or not) to the shareholder's wealth. I stands for the data the bank disposes of: the correlation matrix, its past performance, its objectives. $\Omega$ is a constraint field which take into account regulatory and strategic requirements, and other technical constraints

$$
\Omega=\mathbb{R}_{+}^{M} \bigcap \Omega^{\text {Reg. }} \bigcap \Omega^{\text {Strat. }} \bigcap \mathcal{C}
$$

As said in the previous section, reallocation generates costs we must evaluate and introduce into the model. This could be done by using a penalized objective function or/and by modelling some technical constraints. In the first case, the objective function becomes $f(\alpha ; \mathcal{I})-\zeta g\left(\alpha, \alpha_{0}\right): g$ represents a positive bounded function $\left(g\left(\alpha_{0}, \alpha_{0}\right)=0\right)$ and $\alpha_{0}$ is the current allocation vector. In the second case, technical constraints define a maximal difference between the actual allocation and the solution. Another technical constraint could be introduced in order to take sector competition into account.

We have done a graphical representation of the optimisation problem at the figure (4). This is just an illustration, but the scheme allows us to understand the difference between a traditional asset allocation and a capital allocation problem.

Without any constraint, the solution is generally located on the efficient frontier. Introducing a penalized cost function moves the solution out of the the efficient frontier, to the inside of it. We notice another effect 
due to the objective function: the transformation is usually non linear in respect of the expected return and the risk of the portfolio.

If costs of reallocation are directly captured by the technical constraints, $\Omega$ may become a closed space. In main cases, regulatory requirements introduce a boundary on the right part of the space because they are aimed at limiting risk. We also represent strategic constraints even though, most of the time, strategic constraints cannot be represented by a geometric area on a mean-variance scheme.

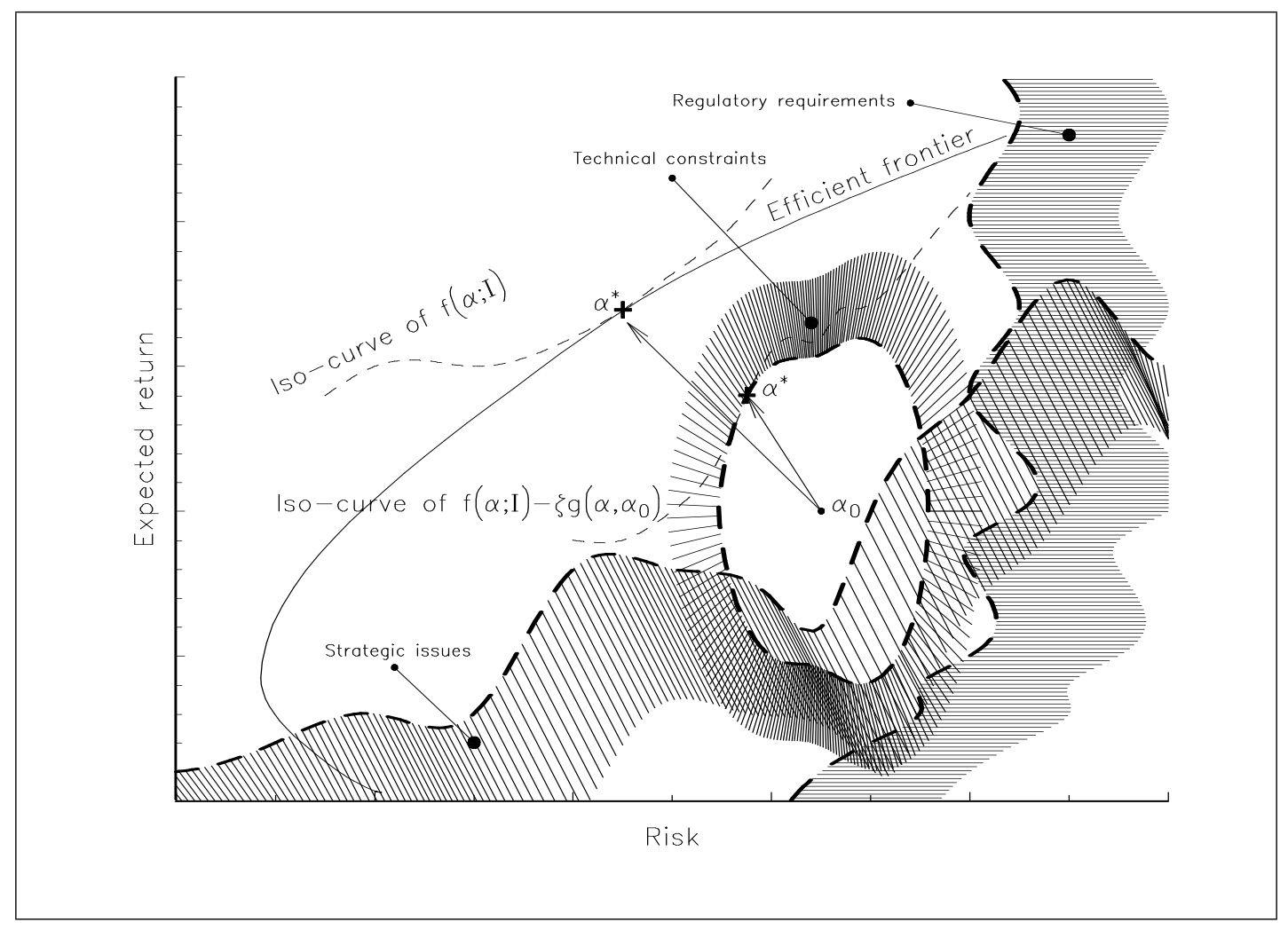

Figure 4: Graphical representation of the optimisation problem

\subsection{An illustration}

In this paragraph, we present some examples illustrating the modelling of capital allocation. For convenience, $\alpha$ denotes the proportion of each business line in the portfolio. Then, we have

$$
\alpha^{\top} \mathbf{1}=1
$$

Let $\tilde{R}$ denote the random vector of the business lines' returns. $\mu$ and $\sigma$ are respectively the vector of the expected returns and the vector of risks. $\rho$ is the correlation matrix of the business lines' returns. The expected return of a portfolio is given by $\alpha^{\top} \mu$ whereas the total risk corresponds to $\sqrt{\alpha^{\top} \Sigma \alpha}$ with

$$
\Sigma_{i, j}=\rho_{i, j} \sigma_{i} \sigma_{j}
$$

Let's point out a small difference with the market factor model presented in the section 2 page 2 . In the internal allocation model, $\sigma_{m}$ is the overall risk of the bank's business line $m$. There is no reason to add any specific risk and furthermore, we assume that $\sigma_{m}$ is a global measure that contains operating risks, etc.

Remark 4 All the computations are done with the values of expected returns and risks obtained previously. 
Let's first introduce a very simple example based on risk-adjusted performance measures (RAPM). PUNJABI [1998] describes these measures as follows:

These measures take into account the risks embedded in the returns as well as the returns themselves and provide a common, aggregated framework to access the contributions of various transactions and business units to the firm's value.

There are different RAPM measures, but the most known (and the leading approach) is RAROC which is extensively documented in the litterature (JAMES [1996] and ZAIK, WALTER, KeLling and JAMES [1996]). RAROC could be viewed as a performance measure defined as follows (STOUGHTON and ZECHNER [1999a]):

$$
\text { RAROC }=\frac{(\text { Expected }) \text { Return minus risk adjustement }}{\text { Economic Capital }}=\varsigma
$$

We suppose that the cost of equity capital is zero so that we have

$$
\varsigma(\alpha)=\frac{\alpha^{\top} \mu}{k_{\beta} \cdot \sqrt{\alpha^{\top} \Sigma \alpha}}
$$

In the equation above, the Economic Capital EC is assimilated to a $V a R$ measure. In a pure gaussian framework with a $99 \%$ confidence level, $k_{\beta}$ is equal to $\left|\Phi^{-1}(0.01)\right|=2,33$. If we do not impose any constraints, the optimisation of $\varsigma(\alpha)$ consists in finding the tangent to the efficient frontier line which cuts the origin as explained in the figure (5).

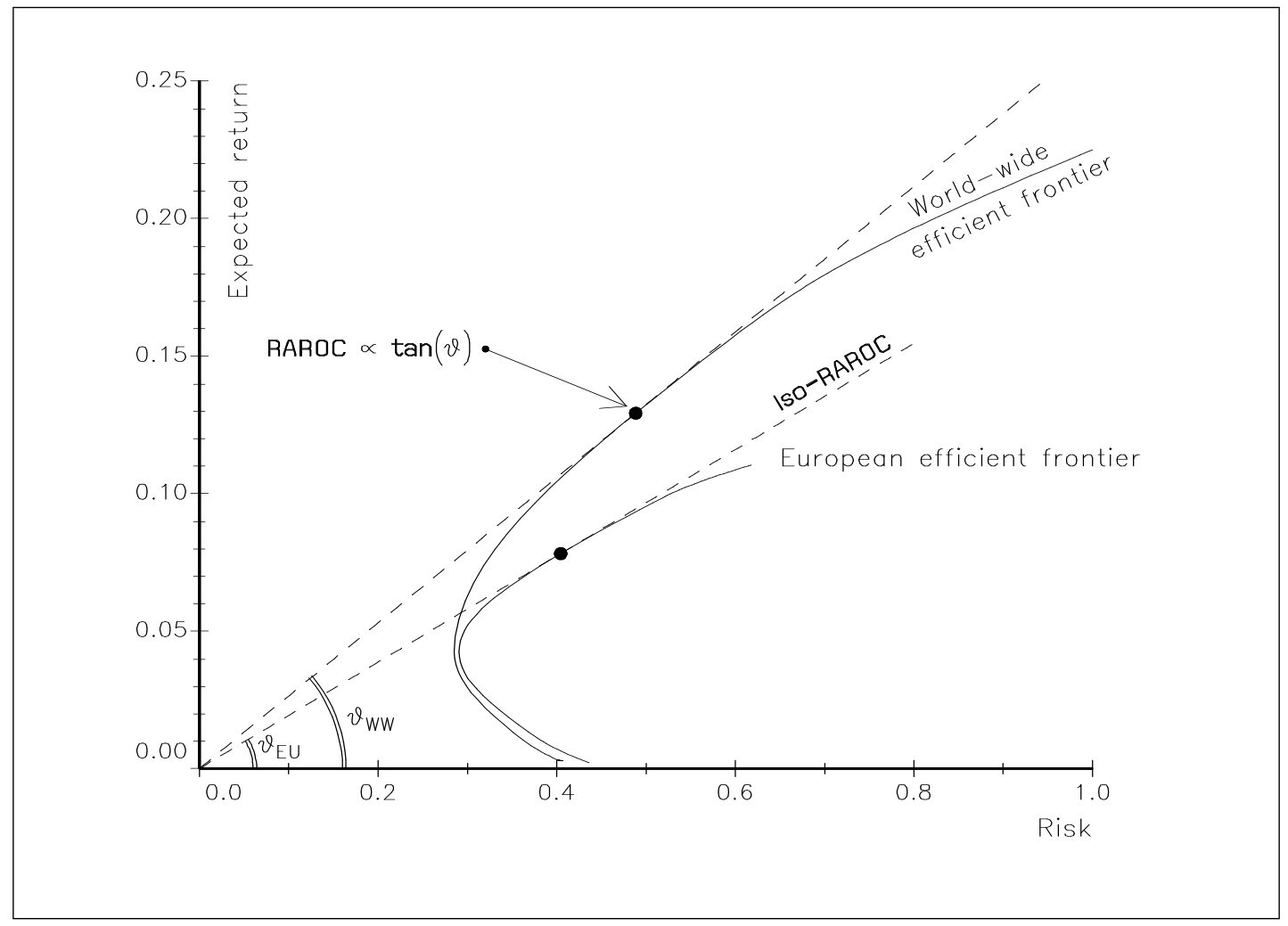

Figure 5: The RAROC problem

Let $\mathbf{C}$ denote the capital. With a non-zero cost of capital $r$, the formula (17) becomes ${ }^{2}$

$$
\varsigma(\alpha)=\frac{\alpha^{\top} \mu-r \mathbf{C}}{k_{\beta} \cdot \sqrt{\alpha^{\top} \Sigma \alpha}}
$$

\footnotetext{
${ }^{2}$ Because of the definition of $\alpha, \mathbf{C}$ is equal to 1 for our example.
} 
The consequence of cost of capital is represented at the figure (6). By analogy to market equilibrium theory, the tangent is the Capital Market Line and the solution of the precedent problem corresponds to the Market Portfolio that has the highest Sharpe Ratio. However, we point out that this market portfolio may be constrained by regulatory requirements on risk. To illustrate this fact, we introduce the restriction $c \sqrt{\alpha^{\top} \Sigma \alpha} \leq C$, which could be interpreted as a $V a R$ constraint. We clearly see that the market portfolio is likely to be unattainable.

Remark 5 The Market Portfolio defined in the previous paragraph is an "internal" portfolio computed from the past performance of the bank's business lines.

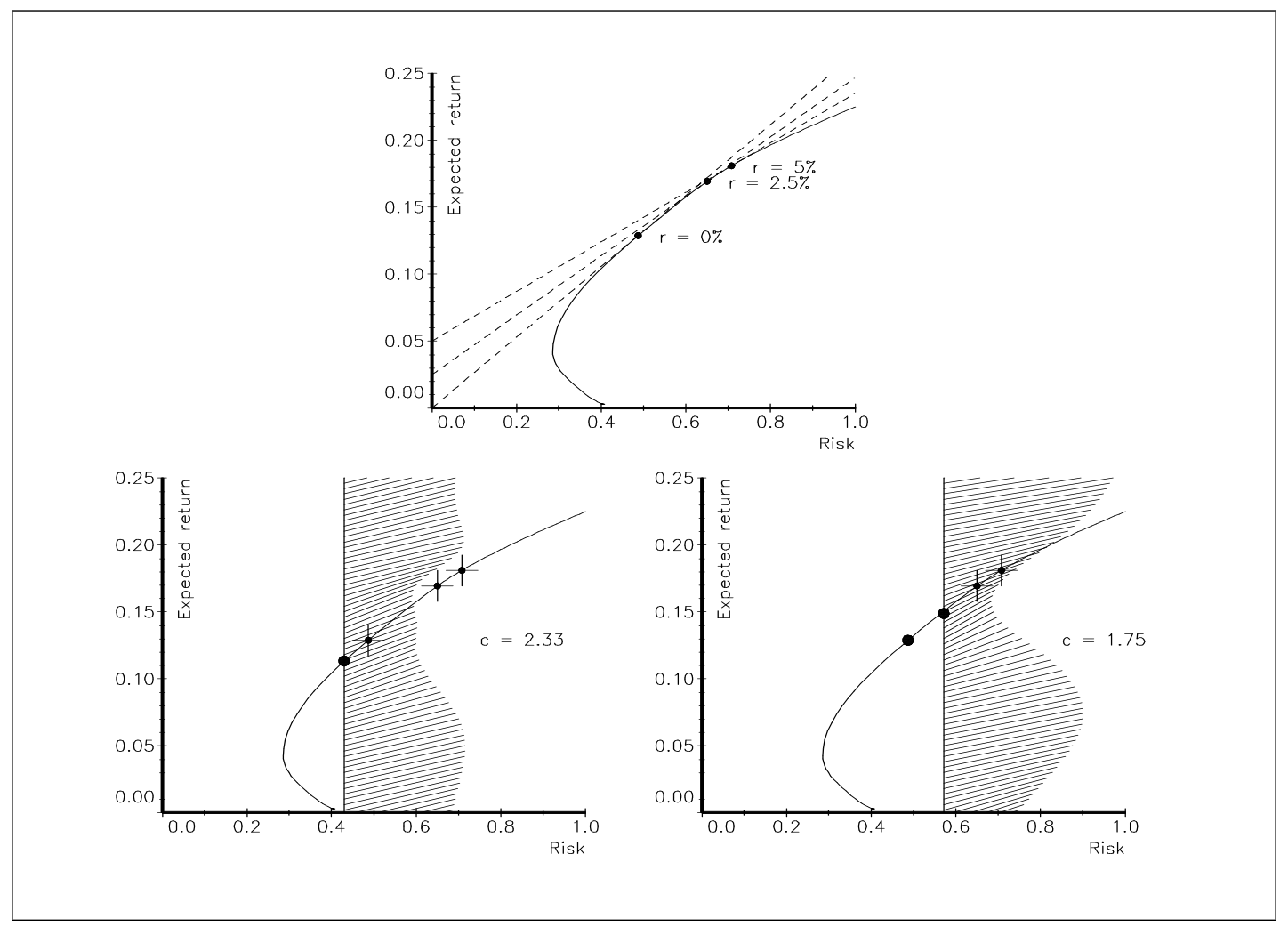

Figure 6: Effect of the cost of capital and regulatory requirements

We now present some examples of reallocation. Let's suppose that the current allocation is as follows:

\begin{tabular}{|l|cccccccc|}
\hline \hline & $\mathrm{BL}$ \#1 & $\mathrm{BL}$ \#2 & $\mathrm{BL}$ \#3 & $\mathrm{BL}$ \#4 & $\mathrm{BL}$ \#5 & $\mathrm{BL}$ \#6 & $\mathrm{BL}$ \#7 & $\mathrm{BL}$ \#8 \\
\hline \hline European Business Lines & $15 \%$ & $15 \%$ & $5 \%$ & $25 \%$ & $10 \%$ & $10 \%$ & & $5 \%$ \\
American Business Lines & & & & $10 \%$ & & $5 \%$ & & \\
\hline Total & $15 \%$ & $15 \%$ & $5 \%$ & $35 \%$ & $10 \%$ & $15 \%$ & $0 \%$ & $5 \%$ \\
\hline \hline
\end{tabular}

If we do not impose any constraint, the solution of the RAROC problem is the market portfolio. Unfortunately, the solution does not depend on the actual allocation. Such a solution is not realistic because it does not take reallocation costs or/and strategic issues into account.

Suppose that the bank managers prefer a "smoothed" reallocation. In a first situation (case I), we impose that each business line share cannot move over $5 \%$. The solution is represented at the figure (7). In a second situtation (case II), we suppose that we consider a geographic reallocation: the amount of each business 


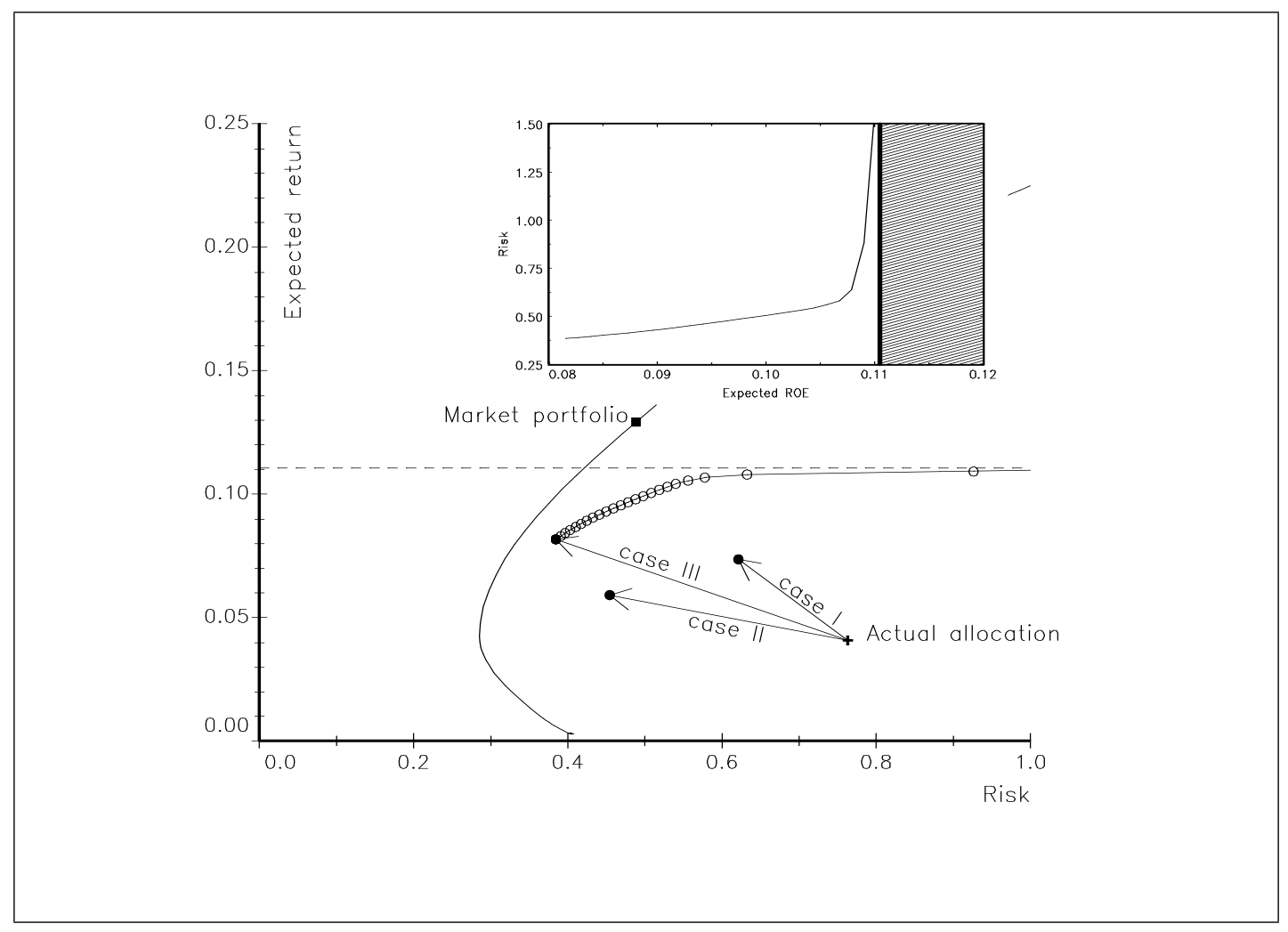

Figure 7: Reallocation examples

line's allocation remains the same but it is apportionned differently among the geographical areas. The case III is the same as the first one, but the move can reach $10 \%$.

Even if the choice of the objective function is not obvious, the main difficulty lies in defining the constraints in a mathematical point of view, in particular strategic ones. Yet this is a very important step which permits to know wether a reallocation policy is feasible or not. Indeed, we must take care that the problem has a solution. Suppose that we impose a target for the expected ROE in the third case ${ }^{3}$. If the target is too high, there is no solution to the problem. In this example, it is very easy to understand and to detect the inconsistency of the constraint. But when the problem becomes more complex, the inconsistency could be more easily detected when the mathemetic translation is done.

\section{Implementing reallocation policy}

The point of the previous section is to determine the optimum but it does not exhibit any path going from the initial allocation to the optimal one. The purpose of this section is to present a very simple method based on the lagrangian multipliers to give reallocation signals. We show that a dynamic policy based on these signals leads to the desired optimum. Implementing such a policy also allows constraints to evolve: the structure of constraints (in particular, strategic and technical constraints) depends on the implemented allocation so that changing the allocation transforms the contraints. The model we develop take this important issue into account.

${ }^{3}$ This could be written as

$$
\frac{\alpha^{\top} \mu}{\mathbf{C}} \geq \mathrm{ROE}_{-}
$$




\subsection{Lagrangian multipliers as marginal prices}

Let's consider the convex optimisation program

$$
\mathcal{P}\left\{\begin{aligned}
\alpha^{\star}= & \arg \max C(\alpha) \\
& \text { s.c. } \alpha \in \Omega
\end{aligned}\right.
$$

where $C(\alpha)$ represents the objective function and $\Omega$ the field of admissible values. We note $\mathcal{P}_{\alpha^{\star}}$ the program value, which means

$$
\mathcal{P}_{\alpha^{\star}}=\sup _{\alpha \in \Omega} C(\alpha)
$$

To simplify the problem, we suppose that $\Omega$ is defined by $I$ inequality constraints:

$$
\Omega=\left\{\alpha \in \mathbb{R}^{M}: g_{i}(\alpha) \leq \gamma_{i}, i \in I\right\}
$$

Remark 6 Equality constraints can be taken into account: a constraint $h(\alpha)=\zeta$ is replaced by the inequality constraints

$$
\left\{\begin{array}{l}
h(\alpha) \leq \zeta \\
-h(\alpha) \leq-\zeta
\end{array}\right.
$$

We assume that the $\mathcal{P}$ problem has a solution (see Demange et Rochet [1992] for existence theorems and unicity ones). In this case, we can prove that there exists a vector $\lambda \in \mathbb{R}^{I}$ satisfying

$$
\left\{\begin{array}{l}
\left.\frac{\partial C(\alpha)}{\partial \alpha}\right|_{\alpha=\alpha^{\star}}=\left.\sum_{i \in I} \lambda_{i} \bullet \frac{\partial g_{i}(\alpha)}{\partial \alpha}\right|_{\alpha=\alpha^{\star}} \\
g_{i}\left(\alpha^{\star}\right) \leq \gamma_{i} \\
\lambda_{i} \geq 0 \\
\lambda_{i}=0 \quad \text { if } \quad g_{i}\left(\alpha^{\star}\right)<\gamma_{i}
\end{array}\right.
$$

$\lambda_{i}$ coefficients are called lagrangian coefficients (or Kühn and Tucker multipliers). Let's point out that these coefficients are always positive. From an economic point of view, they can be interpreted as the marginal prices of the different constraints. A parametrisation $\alpha^{\star}(\gamma)$ (the optimum is associated to the vector $\gamma$ representing the second member of the inequalities) sheds light on this last point. Indeed, we can prove that, for every vector $\varepsilon$ in the vicinity of $\mathbf{0}_{M}$,

$$
\mathcal{P}_{\alpha^{\star}(\gamma+\varepsilon)}=\mathcal{P}_{\alpha^{\star}(\gamma)}+\langle\lambda, \varepsilon\rangle+o(\|\varepsilon\|)
$$

Releasing the $i^{\text {th }}$ constraint by the acquisition of a marginal capacity unit $\varepsilon_{i}$ raises the objective function by $\lambda_{i} \varepsilon_{i}$.

Thus, we can establish wether a reallocation process is profitable or not by comparing $\lambda_{i}$ to this unit's marginal cost of acquisition, and an optimal policy can be implemented so that a constraint is tightened if $\lambda_{i}=0$ or loosened in the other case.

\subsection{An example}

We consider a very simple example based on the previous RAROC problem. We use the allocation of six French banks. The figure (8) represent the expected return and the risk of these six allocation vectors. The table below report the dual prices (or the lagrandian coefficients). We observe that even if two banks are closer in the mean-variance diagram, they may have different dual prices, because their allocation are different. Moreover, the dual prices indicates which allocation to reduce or to increase. 


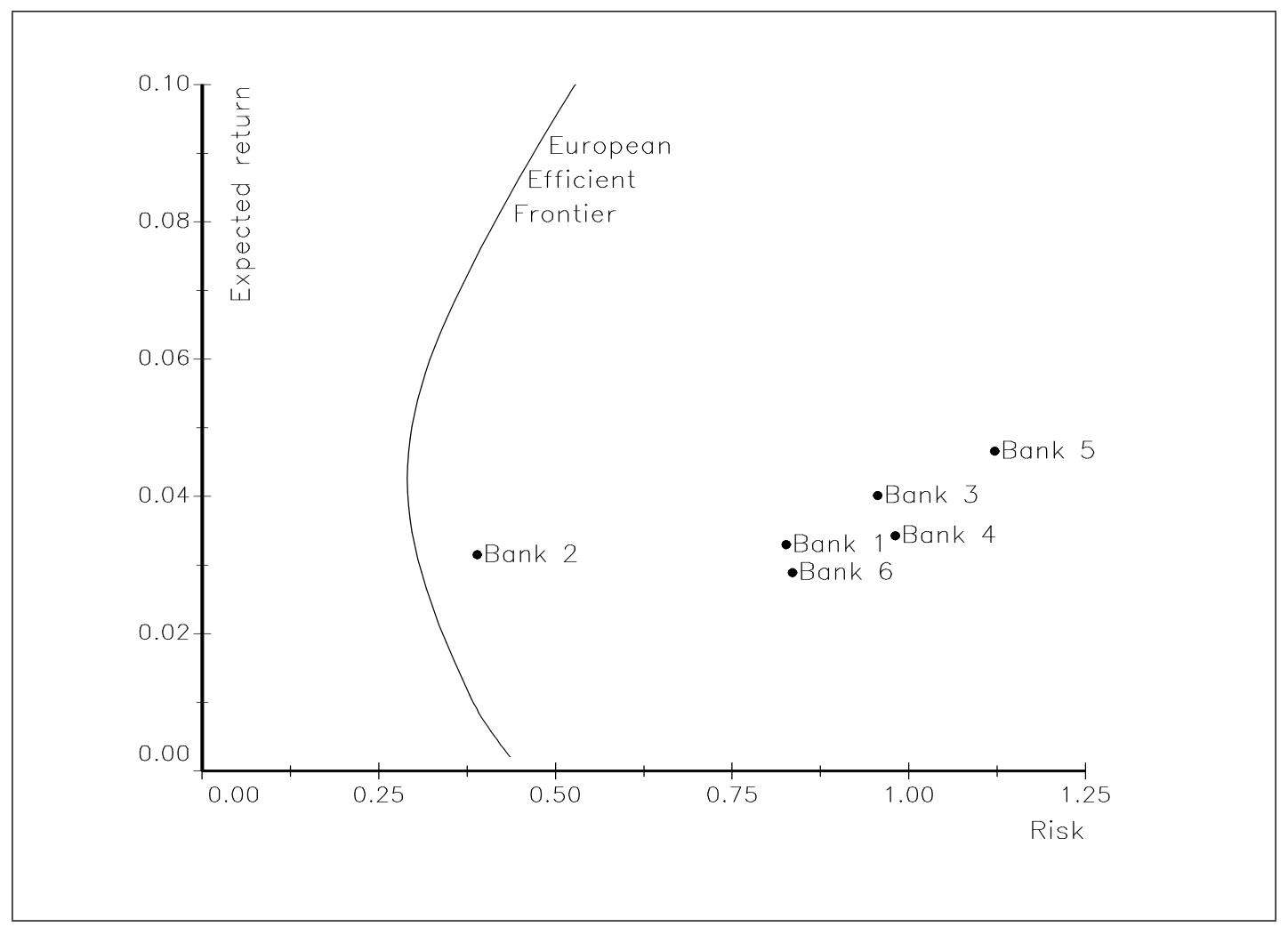

Figure 8: Portfolio performance of the six bank allocations

\begin{tabular}{|c|cc|cc|cc|}
\hline \hline & \multicolumn{2}{|c}{ Bank 1 } & \multicolumn{2}{c}{ Bank 2 } & \multicolumn{2}{c|}{ Bank 3 } \\
\hline \hline & $\alpha_{i}<\dot{\alpha}_{i}$ & $\alpha_{i}>\dot{\alpha}_{i}$ & $\alpha_{i}<\dot{\alpha}_{i}$ & $\alpha_{i}>\dot{\alpha}_{i}$ & $\alpha_{i}<\dot{\alpha}_{i}$ & $\alpha_{i}>\dot{\alpha}_{i}$ \\
\hline \hline BL 1 & & 0.1207 & & 0.2500 & & 0.1023 \\
BL 2 & 0.0042 & & 0.0634 & & 0.0038 & \\
BL 3 & & 0.0712 & & 0.1066 & & 0.0611 \\
BL 4 & 0.0080 & & 0.0606 & & 0.0056 & \\
BL 5 & & 0.0206 & 0.0337 & & & 0.0170 \\
BL 6 & 0.1443 & & 0.5862 & & 0.1349 & \\
BL 7 & 0.0438 & & 0.1936 & & 0.0401 & \\
BL 8 & & 0.0263 & & 0.0194 & & 0.0217 \\
\hline \hline
\end{tabular}

\begin{tabular}{|c|cc|cc|cc|}
\hline \hline & \multicolumn{2}{|c}{ Bank 4 } & \multicolumn{2}{c|}{ Bank 5 } & \multicolumn{2}{c|}{ Bank 6 } \\
\hline \hline & $\alpha_{i}<\dot{\alpha}_{i}$ & $\alpha_{i}>\dot{\alpha}_{i}$ & $\alpha_{i}<\dot{\alpha}_{i}$ & $\alpha_{i}>\dot{\alpha}_{i}$ & $\alpha_{i}<\dot{\alpha}_{i}$ & $\alpha_{i}>\dot{\alpha}_{i}$ \\
\hline \hline BL 1 & & 0.1028 & & 0.0868 & & 0.1221 \\
BL 2 & 0.0009 & & 0.0027 & & 0.0015 & \\
BL 3 & & 0.0622 & & 0.0517 & & 0.0734 \\
BL 4 & 0.0050 & & 0.0046 & & 0.0068 & \\
BL 5 & & 0.0214 & & 0.0137 & & 0.0250 \\
BL 6 & 0.1051 & & 0.1121 & & 0.1203 & \\
BL 7 & 0.0315 & & 0.0330 & & 0.0366 & \\
BL 8 & & 0.0243 & & 0.0186 & & 0.0289 \\
\hline \hline
\end{tabular}




\subsection{Optimum implementation}

The lagrangian multipliers play a very important role in the implementation of a program optimum which, in our case, can be interpreted as a dynamic reallocation exercise. Implementing an optimum amounts to finding a bounded or boundless series $\left\{\alpha^{t}, t \in T\right\}$ satisfying

$$
C\left(\alpha^{t}\right)<C\left(\alpha^{t+1}\right)
$$

and

$$
\left\|\alpha^{T}-\alpha^{\star}\right\|=0
$$

$\left\{\alpha^{t}, t \in T\right\}$ is a path that leads to the optimum. Thus, going from the current situation $\dot{\alpha}$ to the optimal one $\alpha^{\star}$ requires several steps, which seems quite relevant in the case of reallocation. Indeed, a reallocation policy has to be progressive in order to take into account the constraints linked to the current allocation.

Let $\mathcal{P}$ be the convex optimisation problem

$$
\mathcal{P}\left\{\begin{array}{l}
\max C(\alpha) \\
\alpha \in \Omega=\left\{\alpha \in \mathbb{R}^{M}: g_{i}(\alpha) \leq \gamma_{i}, i \in I\right\}
\end{array}\right.
$$

Let $\mathcal{P}^{+}$be the reduced problem

with

$$
\mathcal{P}^{+}\left\{\begin{array}{l}
\max C(\alpha) \\
\alpha \in \Omega \bigcap \mathcal{C}^{-} \bigcap \mathcal{C}^{+}
\end{array}\right.
$$

$$
\begin{aligned}
& \mathcal{C}^{-}=\left\{\alpha \in \mathbb{R}^{M}: \alpha \leq \dot{\alpha}\right\} \\
& \mathcal{C}^{+}=\left\{\alpha \in \mathbb{R}^{M}:-\alpha \leq-\dot{\alpha}\right\}
\end{aligned}
$$

This problem is equivalent to the starting problem with the additional constraint $\alpha=\dot{\alpha}$. Let $\lambda_{m}^{-}$denote the dual price associated with the constraint $\alpha_{m} \leq \dot{\alpha}_{m}$, and $\lambda_{m}^{+}$the price associated with the constraint $\alpha_{m} \geq \dot{\alpha}_{m}$. The optimum $\alpha^{\star}$ of the program $\mathcal{P}$ can be implemented quite easily by applying the equation (24) to the program $\mathcal{P}^{+}$. Indeed, we know that there exists a vector $\varepsilon^{0}$ in the vicinity of $\mathbf{0}_{M}$ satisfying the condition (25) since dual prices are positive or null, and we can define $\alpha^{1}$ as follows

$$
\alpha^{1}=\dot{\alpha}+\varepsilon^{0}
$$

Then, with $\alpha^{1}$ standing in for $\dot{\alpha}$, we can find out another vector $\varepsilon^{1}$ in the vicinity of $\mathbf{0}_{M}$ satisfying the condition (25) and we define the new vector $\alpha^{2}$

$$
\alpha^{2}=\alpha^{1}+\varepsilon^{1}
$$

We reiterate this process until (26) is satisfied. Thus, we obtain a series $\left\{\alpha^{t}, t \in T\right\}$ implementing the optimum.

Remark 7 There exist a large number of series $\left\{\varepsilon^{t}, t \in T\right\}$ based on the equation (24) and satisfying the condition (25). A simple way of choosing a vector $\varepsilon^{t}$ consists in considering the dual prices $\lambda_{m}^{-}$and $\lambda_{m}^{+}$. Let assume for exemple that $\Omega=\mathbb{R}^{M}$. Let $m^{-}$and $m^{+}$be the two business lines associated with the largest dual prices $\lambda_{m}^{-}$et $\lambda_{m}^{+}$

$$
\left\{\begin{array}{l}
m^{-}=\underset{m \in M}{\arg \max } \lambda_{m}^{-} \\
m^{+}=\underset{m \in M}{\arg \max } \lambda_{m}^{+}
\end{array}\right.
$$

Then, we can define $\varepsilon^{t}$ such that

$$
\left\{\begin{array}{l}
\varepsilon_{m}^{t}=0 \quad \text { if } \quad m \in M \wedge\left\{m^{-} ; m^{+}\right\} \\
\varepsilon_{m_{-}}^{t}<0 \\
\varepsilon_{m^{+}}^{t}>0 \\
\varepsilon_{m^{+}}^{t}+\varepsilon_{m_{-}}^{t}=0
\end{array}\right.
$$

It amounts to modifying the allocation vector $\alpha^{t}$ as follows: the business line corresponding to the highest dual price $\lambda_{m}^{+}$is extended while the one corresponding to the highest price $\lambda_{m}^{-}$is reduced. 
We applied the technique above to implement the RAROC solution with $\varepsilon_{m^{+}}^{t}=0.5 \%$. The graphic (9) exhibits the evolution of the portfolio's path in the mean-variance diagram for four initial allocation vectors. Besides, we implemented the following sequential optimisation program in order to compare the results

$$
\left\{\begin{array}{l}
\sup C\left(\alpha^{t+1}\right) \\
\left\|\alpha^{t+1}-\alpha^{t}\right\| \leq \varphi
\end{array}\right.
$$

The graphic (10) exhibits the results in the case of the $\mathcal{L}_{1}$ norm with $\varphi=0.0035$. Let's notice that the path we obtain using the dual prices is quite close to the one we get from the sequential program. Actually, the two paths coincide when variations are infinitesimal $\left(\varphi \longrightarrow 0^{+}\right.$and $\left.\varepsilon_{m_{-}}^{t} \longrightarrow \varepsilon_{m^{+}}^{t}\right)$.

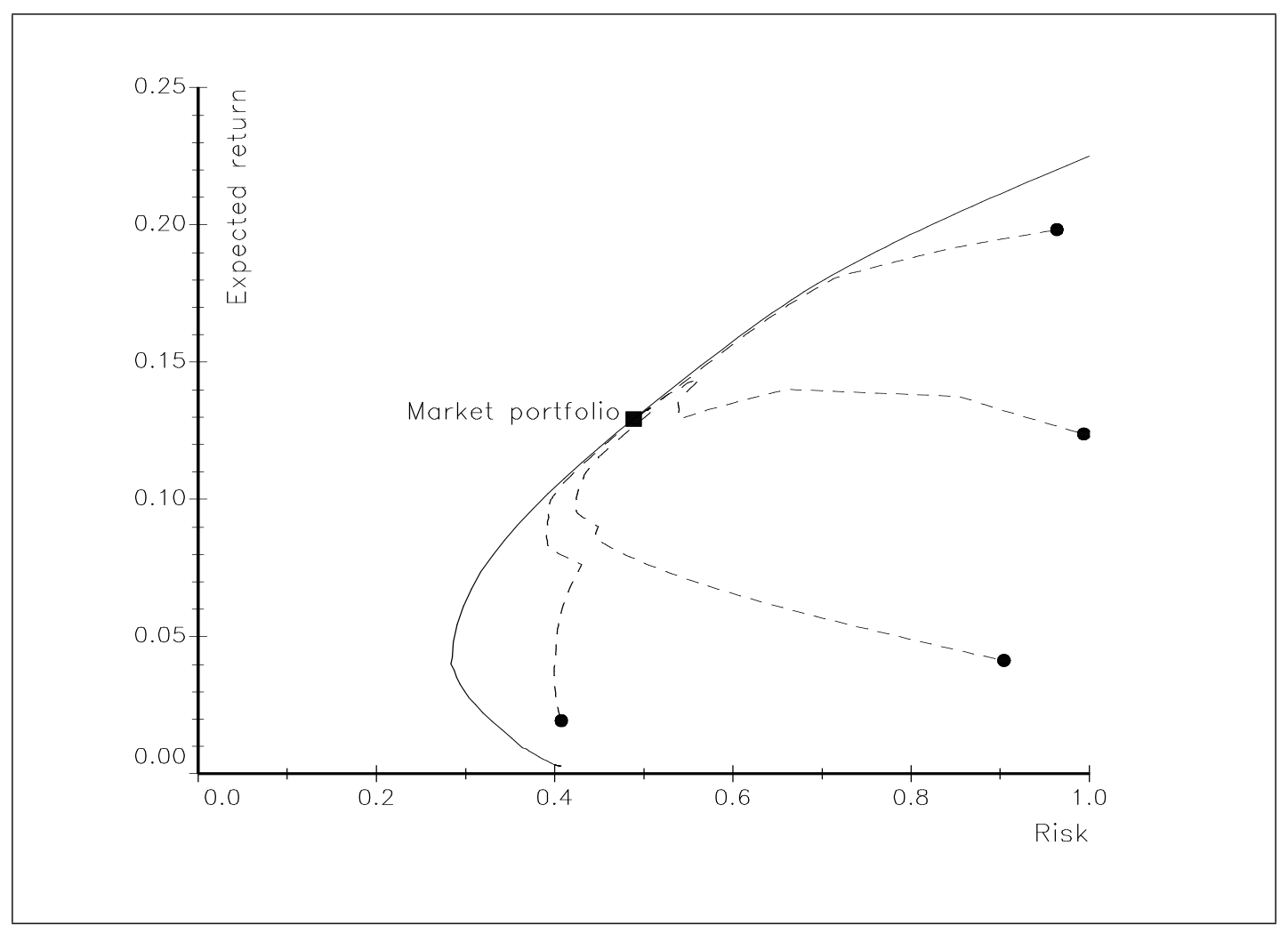

Figure 9: Examples of optimum implementation

In the previous example, we suppose that the parameters and the structure of the constraints always remain the same from one iteration to another. Yet, we may consider that they are likely to evolve. For example, increasing the economic capital of one business line generates some market competition and, as a consequence, reduces the expected return. Therefore, it may be better to consider $\mu$ and $\sigma$ as evolving parameters.

Moreover, moving the allocation may change the structure of the strategic or technical constraints. We could easily take this type of modification into account by modifying the problem at each step $t$

$$
\mathcal{P}^{t} \quad\left\{\begin{array}{l}
\max C^{t}\left(\alpha, \boldsymbol{\Xi}^{t}\right) \\
\alpha \in \Omega^{t}=\left\{\alpha \in \mathbb{R}^{M}: g_{i}^{t}\left(\alpha, \boldsymbol{\Xi}^{t}\right) \leq \gamma_{i}^{t}, i \in I\right\}
\end{array}\right.
$$

$\boldsymbol{\Xi}^{t}$ is defined as a subset of $\left\{\alpha^{0}, \ldots, \alpha^{t-1}\right\}$ :

- If $\boldsymbol{\Xi}^{t}=\emptyset$, then the solution of the program depends neither on the current allocation nor on the portfolio's path. 


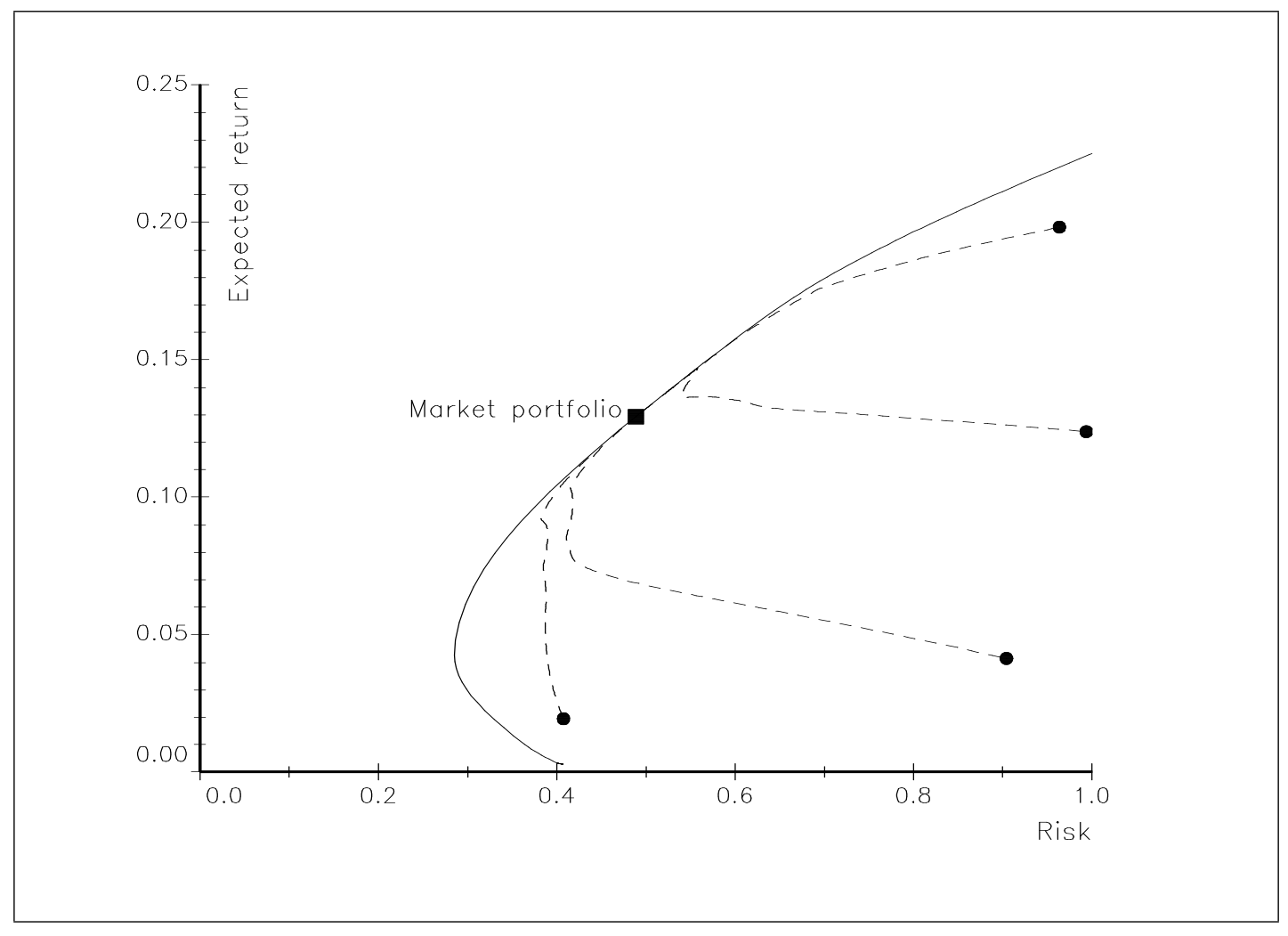

Figure 10: Sequential optimisation with $\mathcal{L}_{1}$ norm

- We could also take $\boldsymbol{\Xi}^{t}=\left\{\alpha^{0}, \ldots, \alpha^{t-1}\right\}$. In this case, the solution depends on the entire path. Implementing an optimum with programs like (35) can be viewed as a scenario analysis and/or hypothesis testing.

\begin{tabular}{|c|c|c|c|c|c|c|c|c|c|}
\hline & & BL \#1 & BL \#2 & BL \#3 & BL \#4 & $\mathrm{BL} \# 5$ & BL \#6 & $\mathrm{BL} \# 7$ & $\mathrm{BL} \# 8$ \\
\hline \multirow[t]{2}{*}{$\alpha^{0}$} & European Business Lines & $15 \%$ & $15 \%$ & $5 \%$ & $25 \%$ & $10 \%$ & $10 \%$ & & $5 \%$ \\
\hline & American Business Lines & & & & $10 \%$ & & $5 \%$ & & \\
\hline \multirow{2}{*}{ (0) } & European Business Lines & $46 \%$ & & $2 \%$ & $19 \%$ & & & & \\
\hline & American Business Lines & & & & & & & & $33 \%$ \\
\hline \multirow{2}{*}{ (1) } & European Business Lines & $43 \%$ & & $9 \%$ & $25 \%$ & $7 \%$ & & & $6 \%$ \\
\hline & American Business Lines & $2 \%$ & $1 \%$ & & $2 \%$ & $1 \%$ & & $1 \%$ & $4 \%$ \\
\hline \multirow{2}{*}{$(2)$} & European Business Lines & $24 \%$ & & $10 \%$ & $24 \%$ & $11 \%$ & & & $6 \%$ \\
\hline & American Business Lines & $5 \%$ & $4 \%$ & $1 \%$ & & $3 \%$ & & $2 \%$ & $10 \%$ \\
\hline \multirow[t]{2}{*}{ (3) } & European Business Lines & $20 \%$ & $16 \%$ & $8 \%$ & $11 \%$ & $12 \%$ & & & $6 \%$ \\
\hline & American Business Lines & $7 \%$ & $2 \%$ & $2 \%$ & & $7 \%$ & & & $9 \%$ \\
\hline
\end{tabular}

The figure (11) illustrates this approach:

0. Given a current allocation, we have computed the portfolio's path and the solution it leads to, without any constraints.

1. Then, we can suppose that because of market limitations, the expected returns depend on the difference between the current allocation and the future one

$$
\mu=\mu\left(\mu^{0}, \alpha^{0}, \alpha\right)
$$

with $\mu^{0}$ the current observed expected return and $\mu<\mu^{0}$ if $\alpha>\alpha^{0}$. 


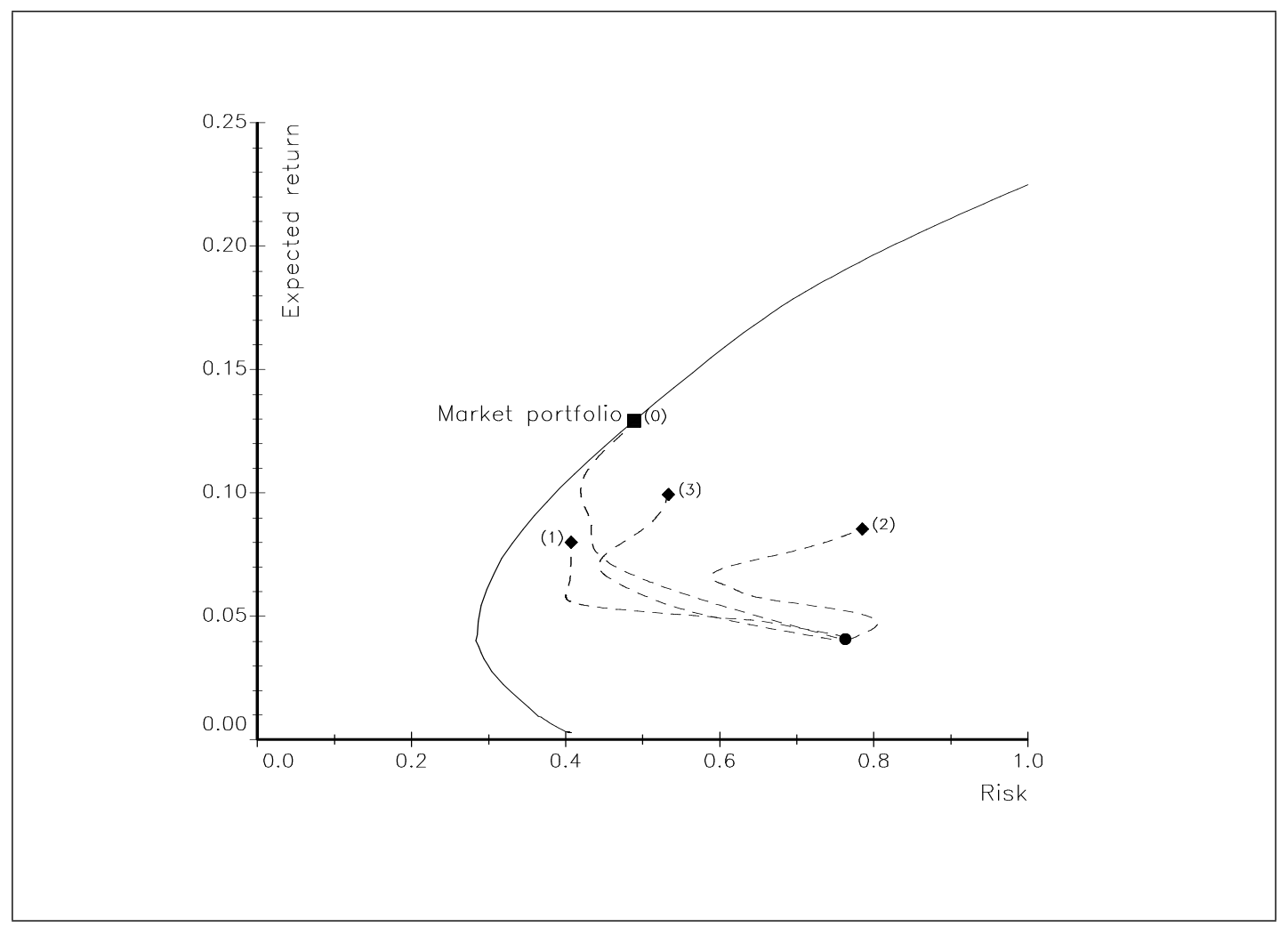

Figure 11: Step-varying optimum implementation

We have considered a numerical example ${ }^{4}$ whose path is reported at the figure (11). In this case, the solution is very different from the Market Portfolio allocation (see the table above).

2. In the program (2), the uncertainty of returns is taken into account when the allocation moves ${ }^{5}$.

3. In a third problem, the objective function $C^{t}$ depends on $\boldsymbol{\Xi}^{t}=\left\{\alpha^{0}, \ldots, \alpha^{t-1}\right\}$. The underlying idea is that reallocation induces costs ${ }^{6}$.

We could do some remarks on these illustrative examples. First, we clearly see that the Market Portfolio is far from the current portfolio. For instance, the American BL \#8 represents 33\% of the Market Portfolio. When we consider reallocation costs, the proportion is reduced to only $9 \%$ (program 3 ). Since the current

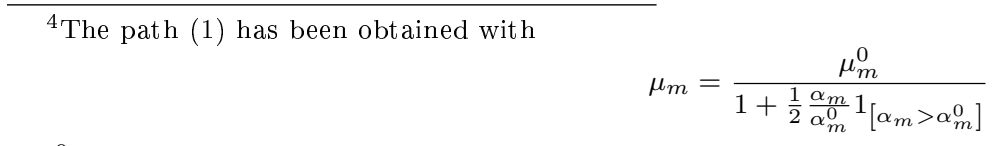

If $\alpha_{m}^{0}$ is equal to zero, we have

$$
\mu_{m}=\frac{\mu_{m}^{0}}{1+200 \alpha_{m}}
$$

${ }^{5}$ The path (2) have been obtained with

$$
\sigma_{m}=\sigma_{m}^{0} 1+10 \alpha_{m}-\alpha_{m}^{0}
$$

${ }^{6}$ The path (3) has been obtained with

$$
C^{t} \quad \alpha, \boldsymbol{\Xi}^{t}=\frac{\alpha^{\top} \mu-r \mathbf{C}-c^{t}}{k_{\beta} \cdot \sqrt{\alpha^{\top} \Sigma \alpha}}
$$

where

$$
c^{t}=\frac{1}{5} \sum_{i=1}^{t-1} \alpha^{i}-\alpha^{i-1}
$$


portfolio does not include this business line, developping this activity implies very huge costs of entry. That's why its proportion becomes very small.

We must point out that we use the ML estimates of expected returns and risks. In an internal approach, it is clear that, for business lines that are not present in the bank, we have to use external data (for example, we could use data provided from benchmarks). These data should be adjusted to take into account the bank's specificity or the bank's strategy. In particular, if the activity $m$ is not represented in the bank, maybe the bank had better leave this activity aside, which means that the bank will set $\mu_{m}=0$.

\section{Concluding remarks}

This paper presents some ideas about bank capital allocation in a top-down approach. The problem is to find out the way a manager must allocate the capital of a bank among different activities. A related issue lies in determining the activities he must enhance and the activities he must reduce, given a current allocation. Such an exercise requires an accurate definition and estimate of the data needed as inputs of the model, In particular we develop a market factor model to estimate the correlation matrix of the different business lines. The allocation problem can be treated as an asset allocation problem with specific constraints. Firstly, the bank's objective has to be clearly defined, which amounts to take into account the shareholder's wealth (for example, RAROC or EVA ${ }^{7}$ ). Secondly the different constraints have to be modelled and finally, the path to reach the solution must be determined.

With allocation models in an Economic Capital framework, we abandon the concept of risk as a volatility measure. Nevertheless, there exist some links between the volatility and the economic capital in a gaussian analysis. In this case, the economic capital can be easily measured with an Earnings-at-Risk method and a $k_{\beta}$ rule. Yet, some problems may occur to reconciliate a top-down approach with a bottom-up one. In the case of our RAROC example, the economic capital of the business line $m$ is $\alpha_{m}$ or $k_{\beta}^{m} \sigma_{m}$. Implicitely, we use different $k_{\beta}$ values to define the solution. That's why we can be confronted with some problems during the capital consumption step (or with a bottom-up approach).

Research into bank capital allocation is heavily developped. At the time, it extensively deals with market risk or credit risk measures (see e.g. [1], [3], [9], [18] and [20]). Two problems are mostly investigated: the measurement problem (see e.g. [5], [6] and [10]) and the estimation method. Research has just begun to cope with two other problems: aggregation (see e.g. [13] and [34]) and allocation programming (see e.g. [11], [27] and [31]). In our top-down approach, we don't face these problems because of the EaR oriented approach and the gaussian analysis we adopted. But it is possible to extend our study and relax these assumptions by using stochastic programming ${ }^{8}$.

\section{References}

[1] Amendment to the capital accord to incorporate market risks, Basle Committee on Banking Supervision, January 1996, $\mathrm{N}^{\circ} \mathbf{2 4}$

[2] Credit Risk Models at Major U.S. Banking Institutions: Current State of the Art and Implications for Assessments of Capital Adequacy, Federal Reserve System Task Force on Internal Credit Risk Models, may 1998

[3] A new capital adequacy framework, Basle Committee on Banking Supervision, June 1999, ${ }^{\circ} 50$

[4] Andersson, F. and S. Uryasev [1999], Credit risk optimisation with conditionnal value-at-risk criterion, Dept. of Industrial and Systems Engineering, University of Florida, Research Report, 99-9

\footnotetext{
${ }^{7}$ In a mathematical point of viewed, the two concepts are closed (see for example Longley-Cook [1998] or Stoughton and Zechner [1999a]). The reader will find an introduction on EVA in Uyemura, Kantor and Pettit [1996].

8 in the spirit of the works of Uryasev ([4], [32]).
} 
[5] Artzner, A., F. Delbaen, J-M. Eber and D. Heath [1997], Thinking coherently, Risk magazine, 10, November, 68-71

[6] Artzner, A., F. Delbaen, J-M. Eber and D. Heath [1999], Coherent measures of risk, Mathematical Finance, 9, 203-228

[7] Baud, N., A. Frachot, P. Martineu and T. Roncalli [1999], Stability of a top-down correlation matrix, Crédit Lyonnais, internal document, 09/29/1999

[8] Belkin, B., L. Forest and S.J. Suchower [1997], Measures of credit risk and loan value in KPMG's LAS $^{\mathrm{SM}}$, Financial Services Consulting-Risk Solutions, KPMG Peat Marwick LLP, october

[9] Credit Suisse Financial Products, CreditRisk+: A Credit Risk Management Framework, Credit Suisse Financial Products, London

[10] Delbaen, F. [1998], Coherent risk measures on general probability spaces, Departement Mathematik, ETH Zürich, Working Paper

[11] Denault, M. [1999], Coherent allocation of risk capital, Swiss Federal Institute of Technology, Working Paper, October

[12] Embrechts, P., Klüppelberg, C. and T. Mikosch [1997], Modelling Extremal Events for Insurance and Finance, Springer-Verlag, Berlin

[13] Embrechts, P., McNeil, A.J. and D. Straumann [1999], Correlation and dependency in risk management : properties and pitfalls, Departement Mathematik, ETH Zürich, Working Paper

[14] FishbuRn, P.C. [1977], Mean-risk analysis with risk associated with below-target returns, American Economic Review, 67, 116-126

[15] Frachot, A., P. Igigabel, P. Martineu and T. Roncalli [1999], Implémentation d'une méthodologie top-down - Estimation d'une matrice de corrélation des rendements d'activités bancaires, Crédit Lyonnais, internal document, 05/15/1999

[16] Froot, K.A. and J.C. Stein [1998a], Risk management, caputal budgeting, and capital structure policy for financial institutions: an integrated approach, Journal of Financial Economics, 47, 55-82

[17] Froot, K.A. and J.C. Stein [1998b], A new approach to capital budgeting for financial institutions, Harvard Business School, Working Paper, July

[18] Gordy, M.B. [1998], A comparative anatomy of credit risk models, Board og Governors of the Federal Reserve System, Working Paper, December

[19] Gouriéroux, C., A. Monfort and A. Trognon [1984], Pseudo maximum likelihood methods: theory, Econometrica, 52, 681-700

[20] Gupton, G.M., C.C. Finger and M. Bhatia [1997], CreditMetrics-Technical Document, J.P. Morgan \& Co. Incorporated, New York

[21] JAmes, C. [1996], RAROC based capital budgeting and performance evaluation: A case study of bank capital allocation, Wharton School, Financial Institutions Center Working Paper, 96-40

[22] Longley-Cook, A.G. [1998], Risk-adjusted economic value analysis, North American Actuarial Journal, 2-1, 87-100

[23] Markowitz, H.M. [1987], Mean-Variance Analyses in Portfolio Choice and Capital Markets, Basil Blackwell, Oxford

[24] Merton, R.C. [1974], On the pricing of corporate debt: the risk structure of interest rates, Journal of Finance, 29, 449-470 
[25] Punjabi, S. [1998], Many happy returns, Risk, June, 71-76

[26] Ross, S.A. [1976], The arbitrage theory of capital asset pricing, Journal of Economic Theory, 13, $341-360$

[27] Schmock, U. and D. Straumann [1999], Allocation of risk capital, RiskLab, Department of Mathematics, ETH Züruch, Working Paper

[28] Sharpe, W.F. [1992], Asset allocation: management style and performance measurement - An asset class factor model can help make order out of chaos, Journal of Portfolio Management, Winter, 7-19

[29] Stoughton, N.M. and J. Zechner [1999a], Optimal capital allocation using RAROC ${ }^{\mathrm{TM}}$ and EVA ${ }^{\circledR}$, University of California Irvine, Working Paper, March

[30] Stoughton, N.M. and J. Zechner [1999b], The dynamics of capital allocation, University of California Irvine, Working Paper, March

[31] Tasche, D. [1999], Risk contributions and performance measurement, Zentrum Mathematik, TU München, Working Paper, July

[32] Uryasev, S. and R.T. Rockafellar [1999], Optimization of conditionnal value-at-risk, Dept. of Industrial and Systems Engineering, University of Florida, Research Report, 99-4

[33] Uyemura, D., C. Kantor and J. Pettit [1996], EVA for banks: Value creation, risk management and profitability measurement, Journal of Applied Corporate Finance, 9, 94-133

[34] WANG, S.S. [1999], Aggegation of correlated risk portfolios: Models \& algorithms, CAS Committee on Theory of Risk, preprint

[35] Zaik, E., J. Walter, G. Kelling and C. James [1996], RAROC at Bank of America: From theory to practice, Journal of Applied Corporate Finance, 9, 83-93 